\title{
Idades e crescimento da cioba, Ocyururs chrysurus, da Costa Central do Brasil
}

\section{(Age and growth of yellowtail snapper, Ocyururs chrysurus, from Central Coast of Brazil)}

\author{
Júlio Neves de Araújo, Agnaldo Silva Martins* \& Karla Gonçalves da Costa \\ Universidade Federal do Espírito Santo \\ Departamento de Ecologia e Recursos Naturais - CCHN \\ (Av. Fernando Ferrari s/n, 29060-900, Vitória, ES, Brasil) \\ *e-mail: agnaldo@npd.ufes.br
}

- Abstract: To age and study the growth of yellowtail snapper, we analyzed readings of otoliths obtained in monthly samplings from commercial landings in the cities of Vitória and Vila Velha, from 1998 to 1999. Marginal increment analysis showed that the formation of a translucent zone occurred in the early fall and during the winter. The observed ages range from 2 to 19 years. Mean back-calculated fork lengths $(F L)$ range from $108 \mathrm{~mm}$ for age 1 to $524 \mathrm{~mm}$ for age 19. The Von Bertalanffy growth equation fitted to the mean back-calculated $F L$ was $F L_{t}=$ $567.1\left(1-e^{\left.0.130(1+0)^{--3}\right)}\right)$. The length-weight relationship was $W=2.68 \times 10^{-5} F L^{2.914}$, where $W=$ whole weight in grams. Yellowtail snapper has a slow growth with annual growth increments in weight raising progressively to the maximum of $164 \mathrm{~g}$ between the 7 and 8 years. The yellowtail snapper has long lifespan and slow growth rates, features of species that are very sensitive to overfishing.

- Resumo: A idade e o crescimento da cioba da costa central do Brasil foram estudados a partir de leituras de otólitos obtidos em amostragens mensais de desembarques comerciais, realizados nas cidades de Vitória e Vila Velha, entre os anos de 1998 e 1999. Através da análise do incremento marginal, foi verificada a formação anual de uma zona translúcida a partir do final do outono e durante o inverno. Foram observadas ciobas de 2 a 19 anos. Os comprimentos furcais $(C F)$ médios retrocalculados variaram de $108 \mathrm{~mm}$ para idade 1 a $524 \mathrm{~mm}$ para idade 19. A equação de Von Bertalanffy ajustada aos $C F$ médios retrocalculados foi $C F_{1}=567,1\left(1-e^{0,130\left(1+00^{--3}\right)}\right)$. A relação comprimento peso foi $P=2,68 \times 10^{-5} C F^{2.914}$, onde $P=$ peso total em gramas. $O$ crescimento é lento, com os incrementos anuais em peso aumentando gradativamente até atingir o máximo de $164 \mathrm{~g}$ entre as idades 7 e 8 anos. Os resultados deste trabalho indicam que a cioba tem longa expectativa de vida e baixas taxas de crescimento somático, características das espécies mais sensiveis à exploração pesqueira.

- Descriptors: Age, Growth, Ocyurus chrysurus, Brazil.

- Descritores: Idades, Crescimento, Ocyurus chrysurus, Brasil.

\section{Introdução}

A cioba, Ocyurus chrysurus, é um lutjanídeo distribuído na costa oeste do Atlântico, desde a Carolina do Norte até o sudeste do Brasil, encontrando-se em maior abundância nas Bahamas, sul da Flórida e no Caribe. Forma grandes cardumes geralmente sobre substratos duros em profundidades entre 10 e 100 metros. Exemplares jovens vivem agrupados em águas litorâneas, geralmente associados a recifes. Os adultos, que podem atingir cerca de 700 $\mathrm{mm}$, ocorrem em águas de plataforma continental interna e externa (Allen, 1985; Manooch \& Drennon, 1987).
Estudos prévios mostraram que a cioba é uma espécie de crescimento lento, vida longa, apresentando grande sobreposição de comprimentos entre as classes etárias (Jonhson, 1983; Manooch \& Drennon, 1987). Manooch (1987) fez uma revisão da literatura sobre determinação de idades e crescimento de lutijanídeos e serranídeos e mostrou que na maior parte dos estudos foi utilizado método de leitura de anéis anuais de crescimento em estruturas de aposição. Este autor concluiu que a aplicação da análise da freqüência de comprimentos é pouco viável, apesar de ser o método mais rápido e de custo reduzido para estudar o crescimento de espécies pertencentes a estas famílias, pois estas não possuem 
as características de crescimento rápido, vida curta e periodo reprodutivo discreto, requeridas para obtenção de resultados confiáveis.

Considerada de boa qualidade para consumo, a cioba é um dos principais recursos pesqueiros demersais na costa central e nordeste do Brasil. Na costa central (entre Salvador e Cabo de São Tomé, 11 a $22^{\circ} \mathrm{S}$ ), é desembarcada principalmente nas cidades de Vitória e Vila Velha pela frota pesqueira de linhade-mão, cujos alvos principais são a cioba, o realito, Rhomboplites aurorubens e o catuá, Cephalopholis filva.

Para se estimar niveis de pesca sustentáveis em sua Zona Econômica Exclusiva (ZEE), o Governo Brasileiro tem conduzido um extenso programa de pesquisa para quantificar as capturas totais, biomassa e estimar os parâmetros populacionais de espécies comerciais e de recursos potenciais. Com esse fim, um programa de amostragem em cruzeiros de pesquisa e amostragens de desembarques comerciais, tem sido conduzido desde 1997. Dentro deste contexto, este trabaho teve como objetivos a determinação de idades e estimativas dos parâmetros de crescimento da cioba da costa central do Brasil.

\section{Material e métodos}

O material utilizado neste trabalho é proveniente da plataforma interna e externa (30 a $100 \mathrm{~m}$ de profundidade) da costa central do Brasil, na área da ZEE, entre as latitudes de 16 e $21^{\circ} 30^{\prime} \mathrm{S}$. A hidrologia da costa central é caracterizada pela presença das águas oligotróficas da corrente do Brasil (Nonaka et. al., 2000). A morfologia do fundo nesta área é muito irregular, sendo em sua maior parte constituida de substrato duro coberto de algas calcárias. A largura da plataforma continental na região estende-se de 8 a $246 \mathrm{~km}$. (Melo el. al. 1975).
Um total de 655 ciobas foram coletadas em amostragens mensais de desembarques da frota comercial de linheiros, realizados na cidade de Vitória entre 1998 e 1999. De cada espécime amostrado, foram registrados, o sexo, o comprimento furcal (CF) em milímetros, medido da extremidade anterior ao centro da forquilha da nadadeira caudal, o peso total $(P)$ em gramas e retirado o par de otólitos sagitta. Para a determinação de idades foram selecionadas até 30 ciobas por classe de comprimento de $10 \mathrm{~mm}$, perfazendo um total de 369 espécimes.

Os otólitos foram incluídos em resina poliéster e cortados numa cortadeira metalográfica de baixa velocidade (Isomet, Buelher). De cada otólito foram retirados dois cortes transversais com espessura aproximada de $0,3 \mathrm{~mm}$. Os cortes foram montados sobre lâminas de vidro, imersos em meio histológico sintético e cobertos com lamínulas. Os otólitos foram cxaminados em lupa, com luz incidente e aumento de 25 vezes. Desta forma foi possivel a distinção de anéis de crescimento opacos e translúcidos alternados (Fig. 1).

As idades foram determinadas como equivalentes ao numero de anéis translúcidos contados. As contagens de anéis dos otólitos foram feitas independentemente por dois "leitores". Un dos leitores, chamado de "primeiro leitor", realizou uma segunda contagem após um período minimo de um mês. Foram considerados legíveis os otólitos para os quais ocorreram pelo menos duas leituras iguais.

Sobre a margem ventral do sulcus acusticus (Fig. 1), foram medidas as distâncias do centro à borda de todos os cortes de otólitos $(R)$, e do centro ao começo de cada anel translúcido $\left(R_{i}\right)$ daqueles considerados legíveis e nos quais a transição entre os anéis foi suficientemente clara. Alguns cortes não foram medidos por não passarem exatamente pelo foco.

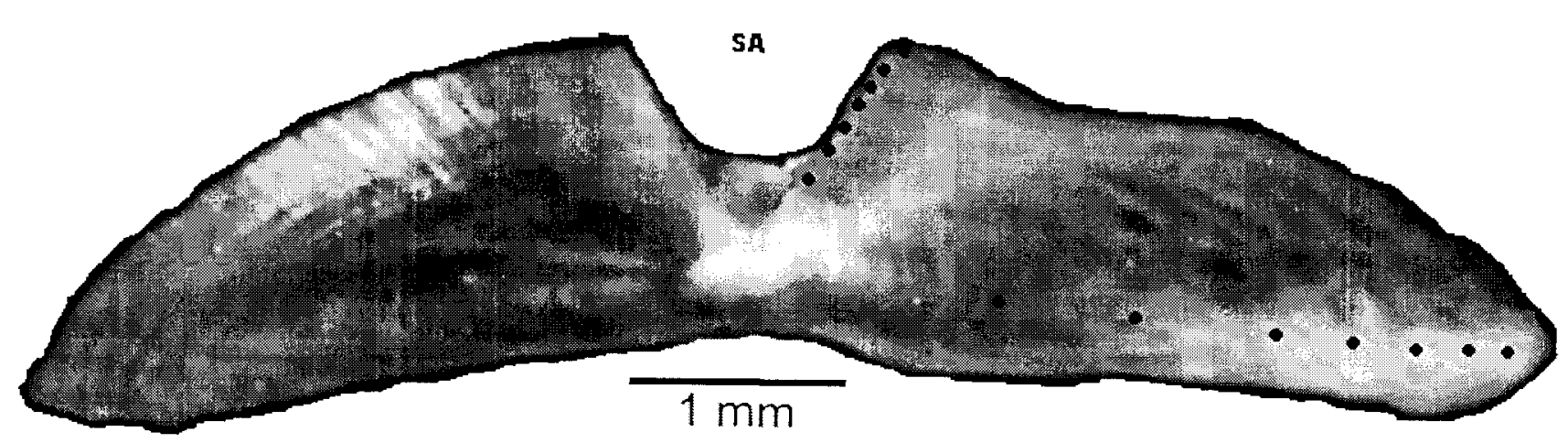

Fig. 1. Conte transversal de um otólito $(0.3 \mathrm{~mm}$ de espessura) observado sob luz refletida. Otólito de uma fêmea de Ocyurus chrysurus com $474 \mathrm{~mm}$ de comprimento fureal e 7 anos de idade, amostrada em selembro de 1998 na costa central do Brasil. 
A periodicidade de formação dos anéis de crescimento nos otólitos foi determinada com a utilização da análise da variação mensal do incremento marginal médio a partir da análise de otólitos de todas as idades. O incremento marginal foi definido como a distância entre o começo do último anel translúcido até a borda do otólito. Os incrementos marginais foram agrupados bimestralmente e comparados mediante o teste nãoparamétrico U de Mann-Whitney.

A relação entre o $C F$ do peixe e o $R$ do otólito, foi descrita pelo modelo linear: $C F=a+b R$, onde $a$ e $b$ são constantes. $O C F$ na época de formação de cada anel translúcido foi retrocalculado pela equação de proporcionalidade corporal para a relação linear: $\left(C F_{i}=\left[\left(a+b R_{i}\right) /(a+b R)\right] C F\right.$, ondo $C F_{i}$ e $R_{i}$ são o comprimento furcal do peixe e o tamanho do otólito correspondentes à idade $i$, respectivamente (Francis, 1990).

Os comprimentos médios retrocalculados foran utilizados para se estimar os parâmetros da equação de crescimento de von Bertalanffy: $C F_{t}=C F_{x}\left(1-e^{k(1-1)}\right)$ onde $C F_{1}$ é o comprimento furcal à idade $t, C F$ o comprimento assintótico, $K$ o coeficiente que representa a velocidade na qual o comprimento do peixe se aproxima do comprimento assintótico e $l_{\text {t }}$ a idade teórica na qual o peixe teria um tamanho nulo se sempre tivesse crescido segundo o modelo proposto. Os parâmetros do modelo foram estimados através do algoritmo de ajuste não-linear Quasi-Newton implementado no programa STATISTICA (StatSoft, Inc., ver 5.l, 1996).

A relação entre o $C F$ e o peso total $(P)$ da cioba foi descrita pelo modelo potencial: $C F=a P^{h}$. onde $a$ e $b$ são constantes.

Os pesos médios por idades foram estimados a partir das equações de crescimento em comprimento e de relação comprimento-peso. As taxas de crescimento em peso foram calculadas como a diferença de peso entre idades sucessivas.

\section{Resultados}

Sobre os cortes examinados observaram-se bandas claras e escuras alternadas, porém com pouco contraste (Fig. 1). Os resultados das comparações de contagens de anéis foram: coincidência entre as leituras do primeiro leitor: $41 \%$; primeira leitura (primeiro leitor) $x$ leitura (segundo leitor): 43\%; segunda leitura (primeiro leitor) $x$ leitura (segundo leitor): $35 \%$; coincidências entre no minimo 2 das 3 leituras: $76 \%$ (281 otólitos).

Foram observados otólitos com 2 a 19 anéis translúcidos, com o maior número de observações para os de 3 a 6 anéis, que representaram aproximadamente $89 \%$ das amostras. Para somente 189 cortes, $51 \%$ do total examinado, todos os anéis foram "claros" o suficiente para se fazer as mediçôes sobre o eixo escolhido.

A correspondência das leituras em otólitos de diferentes idades foi avaliada analisando-se as distribuições de freqüencias dos valores das distâncias entre o centro do otólito e o início do primeiro ao quarto anel translúcido medidas em peixes com 3 a 6 anéis (Fig. 2). Os valores correspondentes a essas medidas foram similares, mostrando consistência entre as leituras em otólitos de diferentes idades. A variação das medidas aumentou para os anéis mais distantes do foco, ocorrendo uma grande sobreposição entre o terceiro e quarto anel.

$O$ incremento marginal médio mensal calculado sobre otólitos de todas as idades (Fig. 3a) apresentou uma tendência decrescente a partir de fevereiro, chegando aos seus valores mínimos entre maio e agosto e voltando a subir após esse período. Os dados bimestrais (Fig. 3b), apresentaram diferenças estatisticamente significativas $(p<0,05)$ entre o terceiro e demais bimestres, excluindo o quarto, e entre o quarto e demais bimestres, excluindo o terceiro. Para os demais casos não foram observadas diferenças estatisticamente significativas. Os baixos valores do incremento marginal nos meses de maio a agosto. indicam que ocorreu a formação anual de um ancl translúcido a partir do final do outono e durante o inverno.

A cioba apresentou uma relação crescente entre o número de anéis de crescimento e o tamanho corporal, mas com uma grande sobreposição de comprimentos entre as classes etárias. A equação que descreveu a relação entre o comprimento e o raio do otólito foi (Fig. 4): ( $F$ $228,51 R+66,148, \mathrm{r}^{2}=0,5539, \mathrm{n}=269$. Os $(\%$ médios retrocalculados à época de formação dos anéis translúcidos variaram de $108 \mathrm{~mm}$ a idade 1 para $524 \mathrm{~mm}$ a idade 19 (Tab. 1). Os CF médios retrocalculados à época de formação do primeiro ao quarto anel em peixes das classes de idades 3 a $8+$ apresentaram uma tendência de aumento em função da idade (Fig. 5). A equação de crescimento de von Bertalanffy estimada a partir dos $C F$ médios retrocalculados foi (Fig. 6): C $F_{1}=567,1\left(1-e^{\text {"1).301 }}\right.$ $(: 0,-3)$ ).

A relação comprimento-peso foi descrita pela equação $P=2,68 \times 10^{-5} C F^{2, \not 1+}\left(r^{2}=0,978, n=\right.$ 655). Os pesos médios por idades e as taxas anuais de crescimento em peso são apresentados na Figura 7. O crescimento é lento, com o incremento anual em peso aumentando de forma gradativa até atingir até o máximo de $164 \mathrm{~g}$ entre as idades 7 e 8 . 

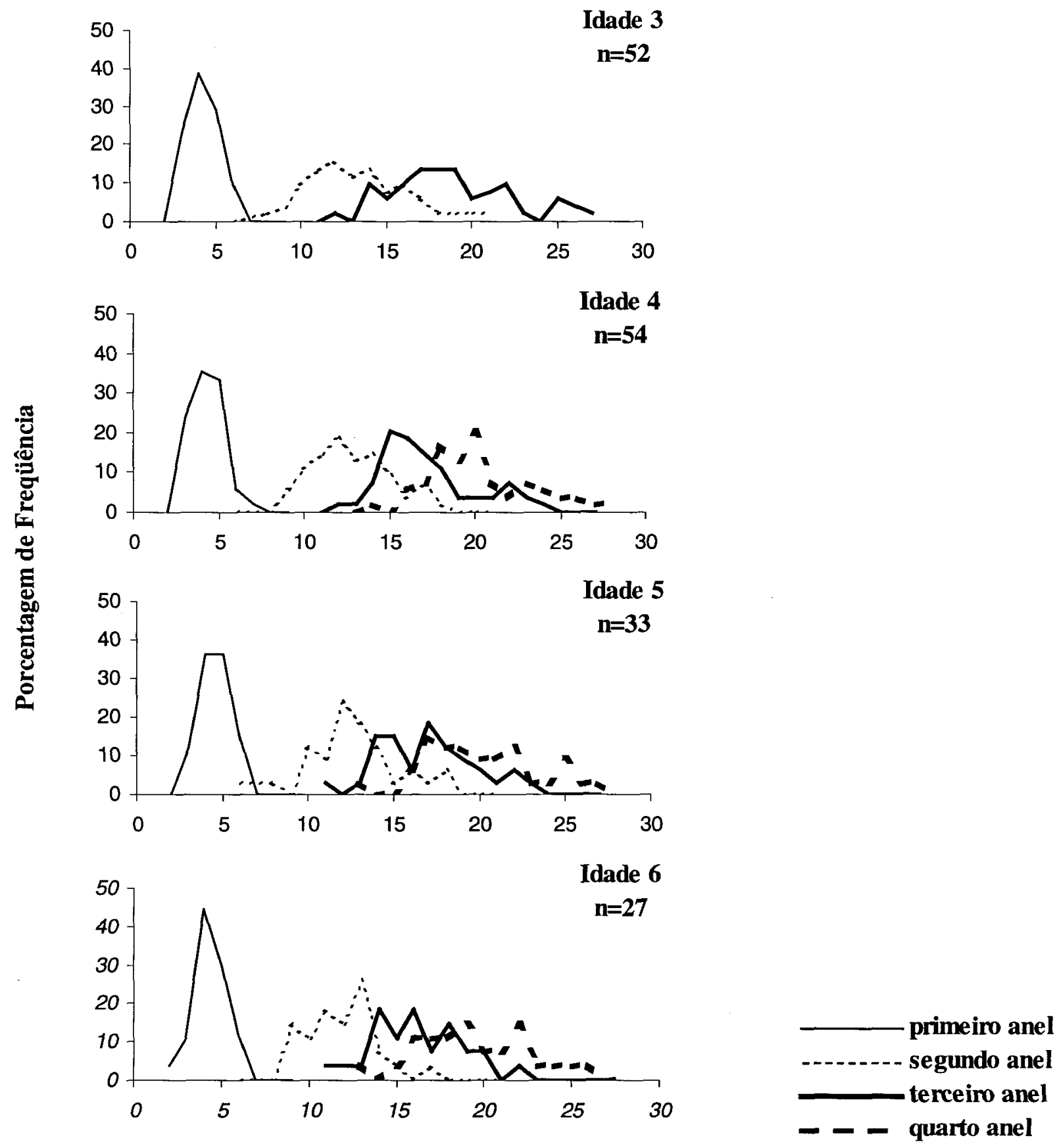

Distância do foco aos anéis (unidade micro. 25x)

Fig. 2. Distribuição de freqüências das medidas correspondentes aos quatro primeiros anéis translúcidos em otólitos de Ocyurus chrysurus de 3 a 6 anos de idade amostrados entre 1998 e 1999 na Costa Central do Brasil. 

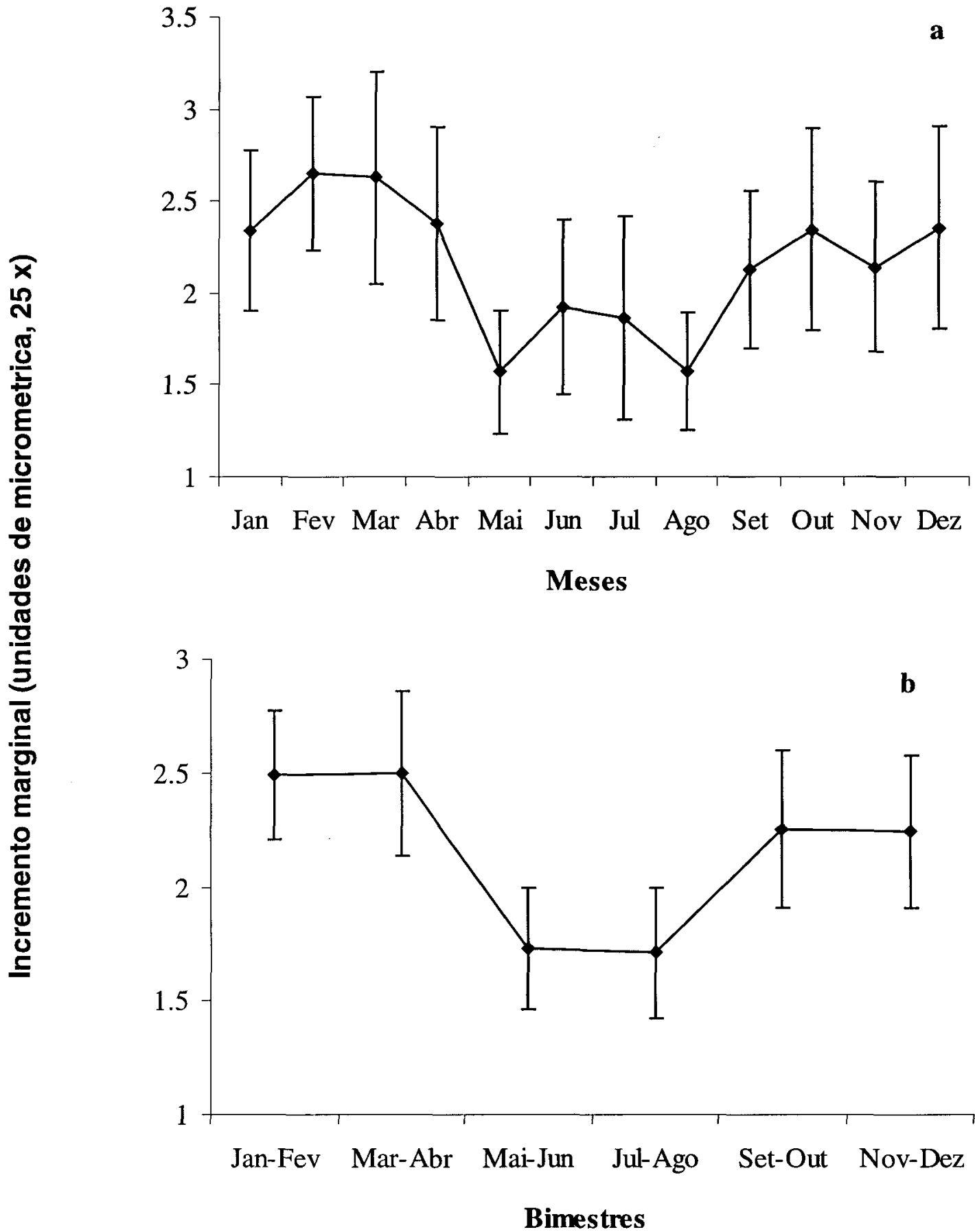

Fig. 3. Médias mensais (a) bimestrais (b) do incremento marginal em anéis de 262 otólitos de Ocyurus chrysurus amostrados entre 1998 e 1999 na costa central do Brasil. Barras verticais: intervalo de confiança $95 \%$. 


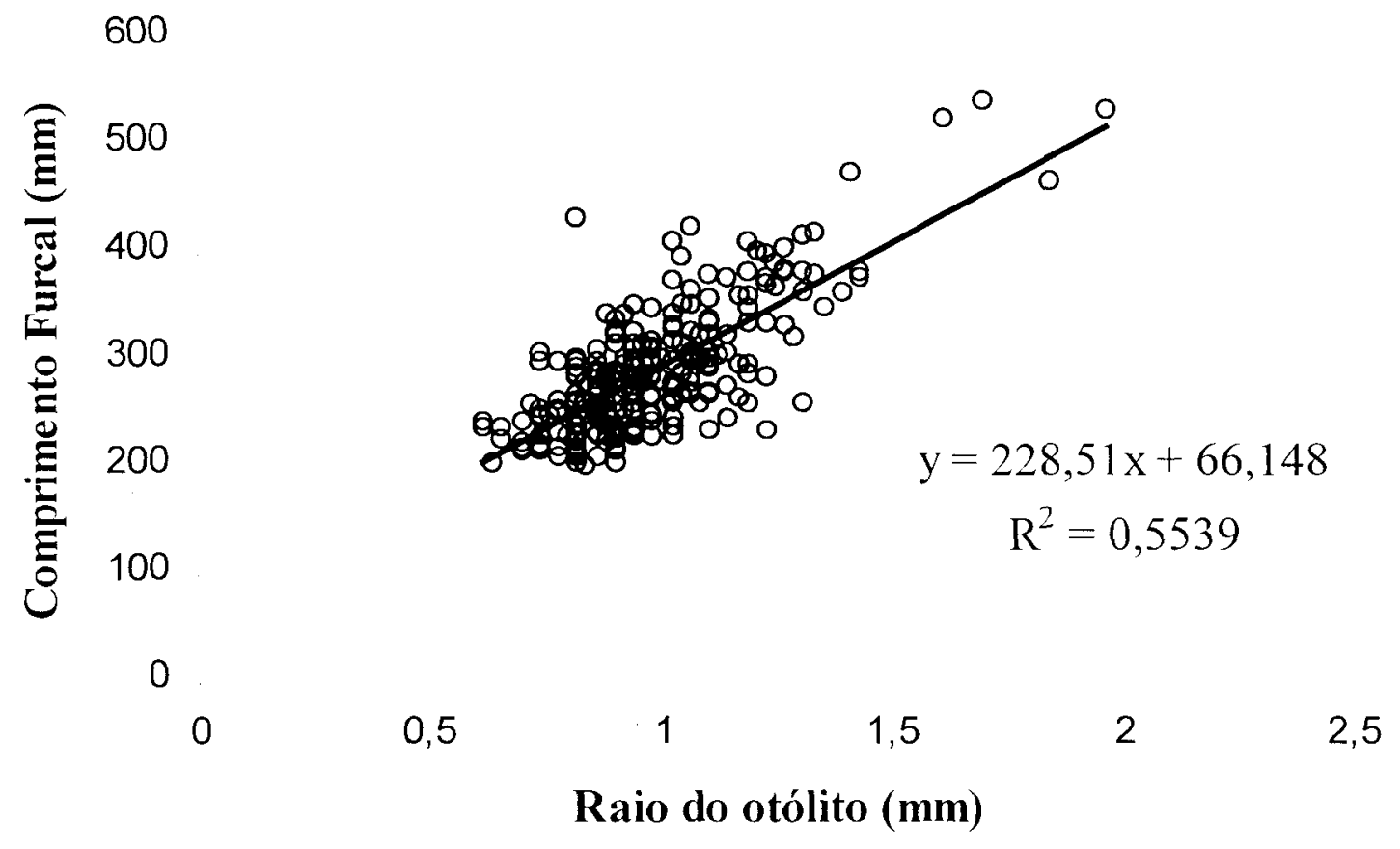

Pig. 4. Relação do comprimento furcal com o raio total dos cortes de otólitos de 269 espécimes de Ocyurus chrysurtus amostrados entre 1998 e 1999 na costa central do Brasil.

Tabela 1. Comprimentos furcais médios ( $\mathrm{mm}$ ) retrocalculados por idades (anos) de 189 espécimes de Ocyurus chrysurus amostrados entre 1997 e 1999 na costa central do Brasil.

\begin{tabular}{|c|c|c|c|c|c|c|c|c|c|c|c|c|c|c|c|c|c|c|c|c|}
\hline $\mathbf{N}$ & Tdade & 1 & 2 & 3 & 4 & 5 & 6 & 7 & 8 & 9 & 10 & 11 & 12 & 13 & 14 & 15 & 16 & 17 & 18 & 19 \\
\hline 2 & 2 & 100 & 187 & & & & & & & & & & & & & & & & & \\
\hline 52 & 3 & 103 & 184 & 232 & & & & & & & & & & & & & & & & \\
\hline 54 & 4 & 107 & 188 & 229 & 258 & & & & & & & & & & & & & & & \\
\hline 33 & 5 & 110 & 188 & 229 & 259 & 283 & & & & & & & & & & & & & & \\
\hline 27 & 6 & 111 & 184 & 226 & 257 & 282 & 302 & & & & & & & & & & & & & \\
\hline 11 & 7 & 112 & 191 & 233 & 268 & 298 & 323 & 346 & & & & & & & & & & & & \\
\hline 3 & 8 & 126 & 192 & 226 & 249 & 272 & 298 & 319 & 343 & & & & & & & & & & & \\
\hline 2 & 9 & 118 & 188 & 225 & 253 & 275 & 299 & 321 & 344 & 363 & & & & & & & & & & \\
\hline 2 & 10 & 132 & 232 & 281 & 319 & 355 & 374 & 396 & 424 & 443 & 462 & & & & & & & & & \\
\hline 1 & 12 & 144 & 189 & 224 & 269 & 314 & 350 & 376 & 399 & 412 & 430 & 439 & 457 & & & & & & & \\
\hline 1 & 14 & 124 & 224 & 269 & 336 & 381 & 403 & 426 & 437 & 454 & 470 & 493 & 509 & 526 & 537 & & & & & \\
\hline \multirow[t]{3}{*}{1} & 19 & 107 & 195 & 243 & 272 & 301 & 325 & 350 & 374 & 379 & 408 & 422 & 437 & 452 & 461 & 476 & 490 & 505 & 515 & 524 \\
\hline & Média & 108 & 187 & 230 & 260 & 288 & 313 & 350 & 377 & 408 & 446 & 451 & 468 & 489 & 499 & 476 & 490 & 505 & 515 & 524 \\
\hline & $N$ & 189 & 189 & 187 & 135 & 81 & 48 & 21 & 10 & 7 & 5 & 3 & 3 & 2 & 2 & 1 & 1 & 1 & 1 & 1 \\
\hline
\end{tabular}




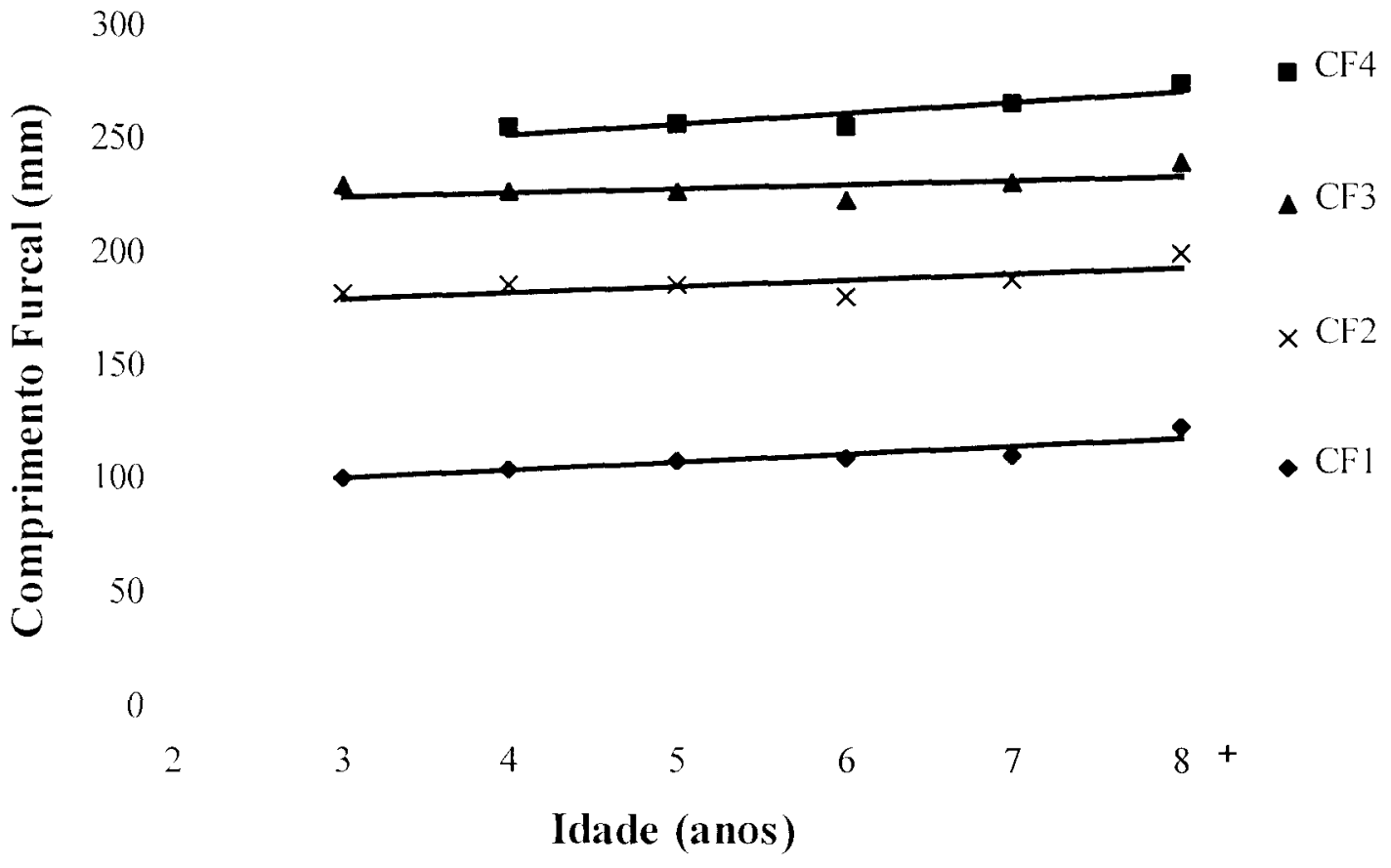

Fig. 5. Comprimentos furcais médios retrocalculados à época de formação do primeiro ao quarto anel translúcido em otólitos de Ocyurus chrysurus das classes de idade 3 a $8+$ amostrados entre 1998 e 1999 na costa central do Brasil. (CFin = Comprimento lurcal correspondente ao ancl $n$ )

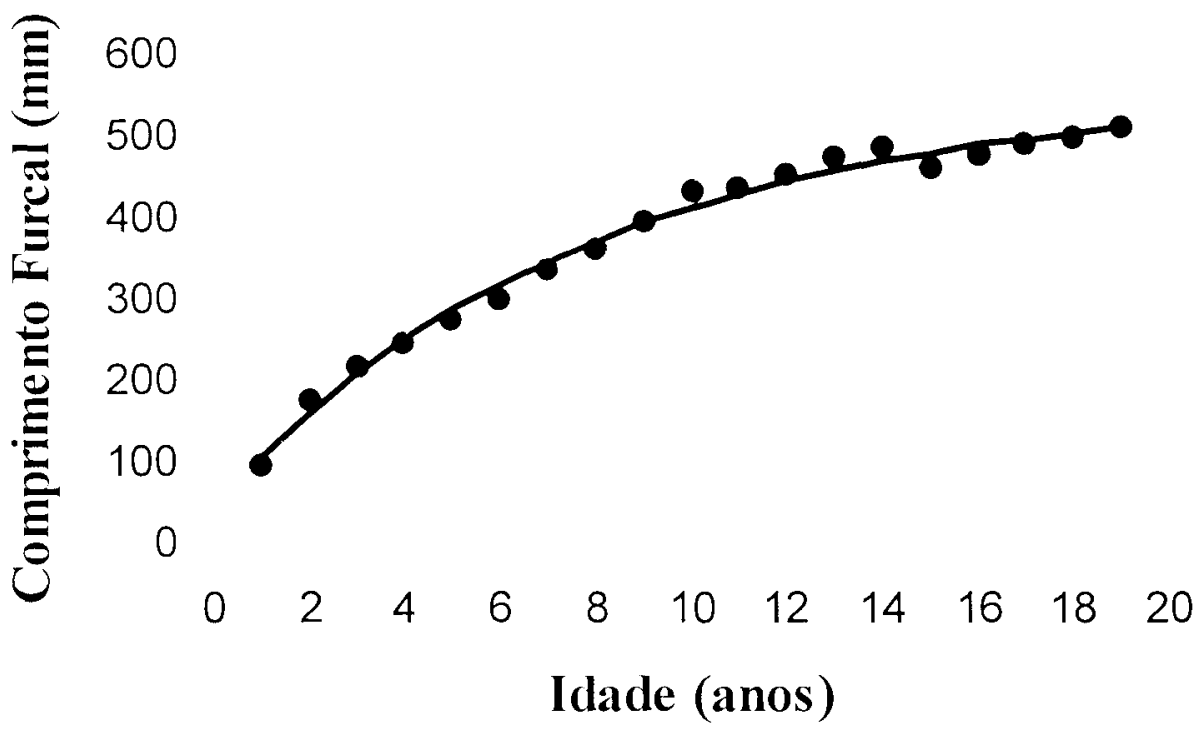

Fig. 6. Curva de crescimento de von Berlalanffy derivada dos comprimentos furcais retrocalculados por idades de Ocyurts chrysurus amostrados entre 1998 e 1999 na costa central do Brasil 


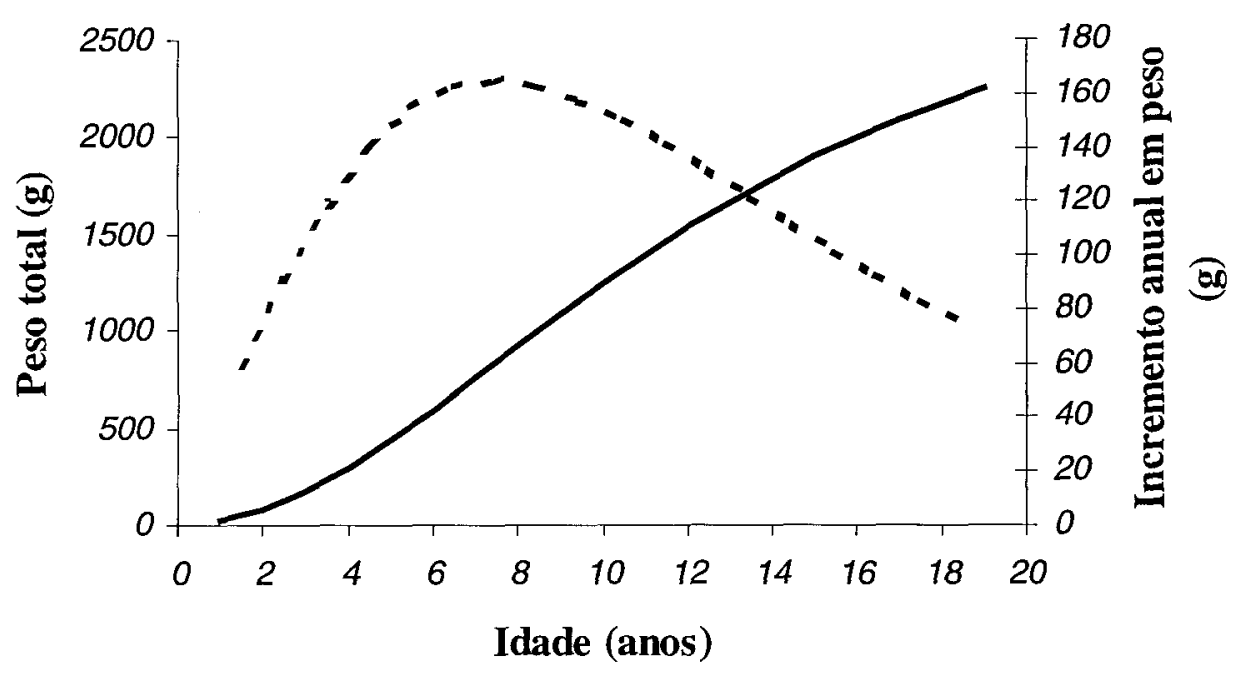

Fig. 7. Curva de crescimento em peso total (linha contínua) e incremento anual em peso (tracejado) por classes de idade, para Ocyurus chrysurus amostrados entre 1998 e 1999 na costa central do Brasil.

\section{Discussão}

Embora tenha sido concluído que os otólitos da cioba podem ser usados para determinação de idades, foi observado pouco contraste entre os anéis opacos e hialinos. A dificuldade de se determinar as idades de peixes tropicais a partir da leitura de anéis de crescimento em estruturas de aposição tem sido relacionada a ausência de grandes variaçōes sazonais nos parâmetros ambientais, combinadas a período reprodutivo extenso e pouca oscilação no crescimento somático (Bagenal \& Tesch, 1978; Manooch 1987). Contudo esta metodologia tem sido empregada em muitos casos (Manooch 1987; Manooch \& Drennon, 1987; Hood \& Schlieder, 1992; Ferreira \& Russ, 1994; Crabtree \& Bullock, 1998).

A formação de anéis de crescimento nos otólitos se relaciona com as mudanças ambientais e metabólicas que os peixes sofrem e que afetam as taxas de calcificação da estrutura. Em geral, os anéis opacos estão relacionados com períodos de crescimento rápido ou altas taxas metabólicas, e se apresentam mais densos devido a uma maior deposição de matéria orgânica. Os anéis translúcidos são relacionados com períodos de crescimento lento e são compostos por uma maior proporção de cálcio e menor de matéria orgânica (Bagenal \& Tesch, 1978; Ferreira \& Russ, 1994). A formação dos anéis translúcidos nos otólitos da cioba ocorreu num período extenso, desde o final do outono até o fim do inverno, provavelmente associado a uma pequena redução da temperatura da água (Nonaka et al., 2000).

Neste trabalho foi observada uma tendência de aumento com a idade dos comprimentos retrocalculados à época de formação de um determinado anel de crescimento, ou seja, o chamado fenômeno de Lee "reverso" (Ricker, 1975). Algumas das possíveis causas para este fenômeno seriam vícios de leitura relacionados com a idade, maior sobrevivência de peixes de crescimento mais rápido nos primeiros anos de vida e vícios relacionados à equação matemática usada para relacionar o comprimento do peixe com o raio do otólito, caso não sejam observados os pressupostos de proporcionalidade entre estas variáveis. A primeira hipótese pode ser considerada pouco provável, pois foi demonstrado haver consistência entre leituras dos otólitos de peixes de diferentes idades (Fig. 2). Portanto, pode ser que o fenômeno de Lee tenha sido decorrente de uma das outras causas, ou mesmo uma combinação de ambas.

Os comprimentos médios retrocalculados por idades para a cioba da costa central do Brasil são comparados aos obtidos em estudos realizados na região do Caribe e Sul da Florida (Tab. 2). Destes, os resultados de Manooch \& Drenon (1987) foram semelhantes aos nossos, inclusive no número de classes etárias observadas, com grande similaridade para os comprimentos retrocalculados para os primeiros seis grupos etários. É de destaque também a 
similaridade dos resultados apresentados por Piedra (1969), que utilizou vértebras no processo de determinação de idades, ainda que tenha observado somentc oito classes etárias. Claro (1983), constatou em Cuba somente cinco classes etárias, cujos comprimentos retrocalculados foram bem maiores do que os observados para a cioba na costa central do Brasil. Johnson (1983) encontrou no sul da Flórida 14 classes de idades. mas com os comprimentos médios retrocalculados para as idades l a 9 sempre superiores aos observados neste estudo.

Na Tabela 3 são comparados os parâmetros de crescimento estimados para a cioba a partir de leitura de anéis de crescimento em estruturas de aposição e de análise de freqüências de comprimentos. Nesta tabela é apresentado também o parâmetro $O^{\prime}$ (Munro \& Pauly, 1983) correspondente a estes estudos. $O O^{\prime}$, chamado de índice de performance de crescimento, é um parâmetro calculado a partir das estimativas do $C_{\infty}$ e do $K$ e pode ser utilizado para se avaliar a "qualidade" das estimativas dos parâmetros crescimento comparandose com estudos anteriores. Segundo Munro \& Pauly (1983), se existe uma série de estimativas do $K$ e do $C_{x}$ para uma determinada espécie, os $O^{\prime}$ correspondentes tenderão a distribuir-se nomalmente em torno de um valor médio. $O{ }^{\prime}$ estimado para cioba da Costa Central do Brasil foi 2,62, diferindo em somente $2,3 \%$ do $O$ ' médio de 2,68 calculado a partir dos valores apresentados na Tabela 3.

Estudos sobre a bionomia de peixes revelaram uma forte relação entre os parâmetros populacionais (Beverton \& Holt, 1959; Pauly, 1980; Jennings el al, 1998; Jemnings et al, 1999). Espécies com ciclo de vida curto, alta taxa de mortalidade natural, maturação precoce, crescimento rápido e pequeno tamanho corporal, tem taxas de crescimento populacional mais rápidas do que espécies de longa vida, crescimento lento, maturação tardia, baixa mortalidade natural e grande tamanho corporal. Portanto, tem sido sugerido o uso de parâmetros populacionais, tais como os de crescimento, para se prever a vulnerabilidade de uma espécie à pesca (Jennings et al, 1999; Jennings, 2000). Assim, os resultados descritos neste e em outros trabalhos, sugerem que a cioba seja muito sensivel aos efeitos da exploração pesqueira, já que é caracterizada por ser uma espécie com ciclo de vida relativamente longo e com baixas taxas de crescimento somático.

Tabela 2. Comparação dos comprimentos furcais médios retrocalculados por idades de Ocyurus chrysurus da costa central do Brasil. sul da Florida e Caribe ( $\Lambda$ daptado de Manooch \& Drennon. 1987).

\begin{tabular}{|c|c|c|c|c|c|c|}
\hline \multirow[t]{2}{*}{ Idade } & \multirow{2}{*}{$\begin{array}{c}\text { Piedra (1969) } \\
\text { Cuba, so }\end{array}$} & \multicolumn{2}{|c|}{ Claro (1983) } & \multirow{2}{*}{$\begin{array}{l}\text { Johnson (1983) } \\
\text { Sul da Flórida }\end{array}$} & \multirow{2}{*}{$\begin{array}{c}\text { Manooch \& Drennon (1987) } \\
\text { Ilhas Virgens e } \\
\text { Porto Rico }\end{array}$} & \multirow{2}{*}{$\begin{array}{c}\text { Araújo elal. } \\
\text { (este estudo) } \\
\text { Costa Central do } \\
\text { Brasil }\end{array}$} \\
\hline & & Cuba, SO & Cuba, NO & & & \\
\hline 1 & 124 & 175 & 162 & 136 & 117 & 108 \\
\hline 2 & 177 & 253 & 248 & 227 & 184 & 187 \\
\hline 3 & 218 & 314 & 316 & 277 & 231 & 230 \\
\hline 4 & 253 & 358 & 358 & 315 & 264 & 260 \\
\hline 5 & 286 & 414 & & 342 & 287 & 288 \\
\hline 6 & 316 & & & 371 & 308 & 313 \\
\hline 7 & 353 & & & 389 & 327 & 350 \\
\hline 8 & 360 & & & 416 & 340 & 377 \\
\hline 9 & & & & 422 & 355 & 408 \\
\hline 10 & & & & 422 & 375 & 446 \\
\hline 11 & & & & 445 & 394 & 451 \\
\hline 12 & & & & 458 & 413 & 468 \\
\hline 13 & & & & 418 & 429 & 489 \\
\hline 14 & & & & 429 & 442 & 499 \\
\hline 15 & & & & & 455 & 476 \\
\hline 16 & & & & & 471 & 490 \\
\hline 17 & & & & & 505 & 505 \\
\hline 18 & & & & & & 515 \\
\hline 19 & & & & & & 524 \\
\hline Estrutura & Vertebras & Otólitos & Otólitos & Otólitos & Otólitos & Otólitos \\
\hline
\end{tabular}


Tabela 3: Parâmetros de crescimento de Ocyurus chrysurus estimados a partir de diferentes métodos de estudo.

\begin{tabular}{|c|c|c|c|c|c|}
\hline$C F_{\infty}(\mathrm{cm})$ & $K(1 / a n o)$ & $t_{0}$ & $\varnothing^{\prime}$ & $\begin{array}{c}\text { Método de } \\
\text { estudo }\end{array}$ & Estudo \\
\hline 53,0 & 0,100 & & 2,45 & escamas & Cantarell, 1982 in: Froese \& Pauly 2001 \\
\hline 45,3 & 0,160 & & 2,52 & escamas & $\begin{array}{l}\text { Mexicano-Cintora e Arregin-Sanchez, } 1989 \text { in: Froese \& } \\
\text { Pauly } 2001\end{array}$ \\
\hline 50,2 & 0,139 & $-0,96$ & 2,54 & otólitos & Manooch \& Drennon, 1987 \\
\hline 60,0 & 0,103 & & 2,57 & otólitos & Rodriguez-Pino, 1962 in: Froese \& Pauly 2001 \\
\hline 50,0 & 0,150 & $-0,80$ & 2,57 & vèrtebras & Piedra, 1969 in Mannoch \& Drennon, 1987 \\
\hline 56,7 & 0,129 & $-0,77$ & 2,62 & otólitos & Araujo et al., este estudo \\
\hline 69,3 & 0,100 & $-1,69$ & 2,68 & otólitos & $\begin{array}{l}\text { Carillo de Albornoz \& Ramiro, } 1968 \text { in: Froese \& Pauly } \\
2001\end{array}$ \\
\hline 41,0 & 0.290 & & 2,69 & $\begin{array}{l}\text { freqüencias de } \\
\text { comprimentos }\end{array}$ & Perez e Rubio, 1986 in: Froese \& Pauly 2001 \\
\hline 45,0 & 0,279 & $-0,36$ & 2,75 & otólitos & Johnson, 1983 in: Mannoch e Drennon, 1987 \\
\hline 49,2 & 0,260 & $-1,19$ & 2,80 & não definido & Piedra, 1965 in: Froese \& Pauly 2001 \\
\hline 68,0 & 0,159 & $-0,85$ & 2,87 & otólitos & Claro, 1983 in: Mannoch \& Drennon, 1987 \\
\hline 47,3 & 0,332 & $-0,27$ & 2,87 & otólitos & Claro, 1983 in: Froese \& Pauly 2001 \\
\hline 60,0 & 0,250 & & 2,95 & $\begin{array}{l}\text { freqüências de } \\
\text { comprimentos }\end{array}$ & Thompson e Munro, 1983 in: Froese \& Pauly \\
\hline
\end{tabular}

\section{Referências bibliográficas}

Allen, G. R. 1985. Snappers of the world. An annotated and illustrated catalogue of lutjanid species known to date. FAO Fish. Synop., (125): 1-208 p.

Bagenal, T. B. \& Tesch, F. W. 1978. Age and growth. In: Bagenal, T. B. ed. Methods for assessment of fish production in fiesh waters. Oxford, Blackwell Scientific Publ., p.101-136.

Beverton, R. J. H. \& Holt, S. J. 1959. A review of the lifespans and mortality rates of fish in nature, and their relation to growth and other physiological characteristics. In: Wolstenholme, G. E. \& O'Connor, M. eds. CIBA Foundation Collegnia on Ageing: the lifespan of animals. London, Churchill Ltd, 5:142-180.
Cantarell, E. 1982. Determinación de la edad y el ritmo de crecimiento del pargo canané Ocyurus chrysurus, (Bloch 1871), en el estado de Yucatán. Tesis de Licenciatura. México, UNAM, E.N.E.P.I.

Carrillo de Albornoz, C. \& Ramiro. M. E. 1988 Estudio biológico de la rabirrubia, Ocyuru chrysurus, en el W. de la plataforma SE de Cuba I. Edad y crecimiento. Revta Investnes mar. Cuba, 9(1):9-24.

Claro, R. 1983. Ecologia y ciclo de vida de la rabirrubia, Ocyurus chrysurus (Bloch), en la plataforma Cubana II. Edad y crecimiento. estructura de poblaciones y pesquerias. Rep. Invest. Inst. Oceanol. Acad. Cienc., Cuba, 19:133. 
Crabtree, R. E. \& Bullock, L. H. 1998. Age, growth and reproduction of black grouper, Mycteroperca bonaci, in Florida waters. Fish. Bull., 96(4):735753.

Ferreira, B. P. \& Russ, G. R. 1994. Age validation and estimation of growth rate of the coral trout, Plectropomus leopardus, (Lacepede 1802) from Lizard Island, Northem Great Barrier Reef. Fish. Bull., 92(1): 46-57.

Francis, R. I. C. C. 1990 . Back-calculation of fish length: a critical review. J. Fish Biol. 36(6):883992.

llood, P. B. \& Schlieder, R. A. 1992. Age, growth and reproduction of gag, Mycteroperea microlepis (Pisces: Serranidae), in the eastern Gulf of Mexico. Bull. Mar. Sci., 51(3):337-352.

Jemnings, S. 2000. Patterns and prediction of population recovery in marine reserves. Rev. Fish Biol. Fish., 10(2):209-231.

Jemnings, S.; Reynolds, J. D. \& Mills, S. C. 1998. Life history correlates of responses to fisheries exploitation. Proc. R. Soc. Biol. Sci., 265(1393):333-339.

Jennings, S.; Reynolds, J. D. \& Polunin, N. V. C. 1999. Predicting the vulnerability of tropical reef fishes to exploitation with phylogenies and life histories. Conserv. Biol., 13(6): 1466-1475.

Johnson, A. G. 1983. Age and growth of yellowtail snapper from South Florida. Trans. Am. Fish. Soc., 112:173-177.

Manooch III, C. S. 1987. Age and growth of snappers and groupers. In: Polovina, J. J. \& Ralston, S. eds Tropical snapper and groupers: biology and fisheries management. Boulder, Westview Press, p.329-373.

Manooch IlI, C. S. \& Dremnon, C. L. 1987. Age and growth of yellowtail snapper and queen triggerfish collected from the U. S. Virgin Islands and Puerto Rico. Fish. Res., 6(1):53-68

Melo, U.; Summerhayes, C. P. \& Ellis, J. P. 1975. Upper continental margin sedimentation off Brazil. Part. IV. Salvador to Vitoria, Southeastern Brazil. Contr. Sedimentol., 4:78-116.
Mexicano-Cintora, G. \& Arreguin-Sánchez, F. 1989. Estimación de edad y crecimiento del pargo canané, Ocyurus chrysurus, del litoral de Yucatán, México. México. Centro de Investigación y Estudios Avanzados del Instituto Politécnico Nacional.

Munro, J. L. \& Pauly, D. 1983. A simple method for comparing growth of fishes and invertebrates. ICLARM Fishbyte, I(1):5-6.

Nonaka, R. H.; Matsuura, Y. \& Suzuki, K. 2000. Seasonal variation in larval fish assemblages in relation to oceanographic conditions in the Abrolhos Bank region off eastern Brazil. Fish. Bull., 98(4):767-784

Pauly, D. 1980. On the interrelationships between natural mortality, growth parameters, and mean environmental temperature in 175 fish stocks. J. Cons. int. Explor. Mer., 39:175-192.

Perez. A. \& Rubio, R. 1986. Análisis electrónico de frequencias de largo en poblaciones de peces cubanos. Foro Cient. In: Forum Cientifico del CIP, 5. Havana, 1986.

Piedra, G. 1965. Materiales sobre la biologia de la rabirrubia, Ocyurus chrysurus (Bloch). Invest. Pesq. Soviet-Cubanas Pischevaia Promishlennost, 1:267-283.

Piedra, G. 1969. Materials on the biology of the yellow-tail snapper, Ocyurus chrysurus (Bloch). Soviet. Cuban Fish. Res., 4:251-269

Ricker, W. E. 1975. Computation and interpretation of biological statistics of fish populations. Bull Fish. Res. Bd. Can., 191:382p.

Rodriguez Pino, Z. 1962. Estudios estadisticos y biologicos sobre la biajaiba Lutjanus synagris. Centro de Investigaciones Pesqueras. Nota sobre Investigaciones, (4):99 $\mathrm{p}$.

Thompson, R. \& Munro, J. L. 1983. The biology, ecology and bionomics of Caribbean reef fishes: Lutjanidae (snappers). In: Munro, J. L. ed. Caribbean coral reef fishery resources. ICLARM Stud. Ver., 7:94-109.

(Mamuscrito recebido 05 outubro 2001; revisado 08 outubro 2002; aceito 29 outubro 2002) 